\title{
Donor/Acceptor Properties of Purine and Pyrimidine Bases
}

\section{Nataliya Obernikhina ${ }^{1 *}$, Marina Kachaeva ${ }^{2}$, Volodymir Shchodryi ${ }^{3}$, Yaroslav Prostota ${ }^{2}$, Oleksiy Kachkovsky ${ }^{2}$, Vladimir Brovarets ${ }^{2}$ and Zenoviy Tkachuk ${ }^{3}$}

\author{
${ }^{1}$ Bogomolets National Medical University, Department of Bioorganic and Biological Chemistry, \\ 34 Peremogy Ave., 03055, Kyiv, Ukraine \\ ${ }^{2}$ Institute of Bioorganic Chemistry and Petrochemistry NASU, 1 Murmanskaya Str., 02660, Kyiv, \\ Ukraine; marinaka4aeva@gmail.com (M.K.); prostotayaroslav@hotmail.com (Y.P.); \\ kachkovskyalexey@gmail.com (O.K.); Brovarets@gmail.com (V.B.) \\ ${ }^{3}$ Institute of Molecular Biology and Genetics NASU, 150 Zabolotnogo Str., 03143, Kyiv, Ukraine; \\ shodryj1992@gmail.com (V.S.); ztkachuk@bigmir.net (Z.T.)
}

*Corresponding author: nataliya.obernikhina@nmu.ua

\begin{abstract}
We have studied the relative positions of the frontier levels determine the main electronic properties and reaction ability, there is important necessity to compare the MO of the purine and pyrimidine bases: adenine $(A)$, guanine $(\mathrm{G})$, cytosine $(\mathrm{C})$, thymine $(\mathrm{T})$ and uracil $(\mathrm{U})$. The donor and acceptor properties are the fundamental characteristic of the conjugated molecules and can be quantitatively estimated by relative positions of the frontier molecular orbitals. The MO's energies can be estimated experimentally or quantum-chemically. Analysis of the relative position of the frontier levels (calibrated by the energy gap) enables the investigation of the donor/acceptor properties of the RNA/DNA bases more detailed. The index $\varphi 0$ is proposed for the quantitative quantum-chemical estimation of the donor ability of the conjugated molecules: it points on the shifting of the energy gap relative to the reference electron balanced system. The RNA/DNA bases divided strictly by two groups: predominantly donors $(\varphi 0>0.5)$ and predominantly acceptors $(\varphi 0>0.5)$. Each representative base of the first group forms the stable base pair the representative base of the second group the difference of indices $\Delta \varphi 0$ should be optimal to enables the DNA replication.
\end{abstract}

Keywords: purine and pyrimidine bases; donor/acceptor properties; quantum-chemical calculations; frontier levels; middle energy gap; parameter $\varphi$ 


\section{Introduction}

The nucleic bases - adenine, thymine, guanine, cytosine and uracil are wide known building blocks of deoxyribonucleic acid (DNA) and ribonucleic acid (RNA). A determination of their electronic structure is a great interest so as the NA bases are exceptional stable complex molecular assembly with respect to exposure of various environmental factors (Zinger, 1984). It is well known that NA bases can generate the long chain owing to formation of the hydrogen bonding between pair of NA bases; such H-bonding is established to be main type of the intermolecular and intramolecular interactions which are responsible to biological process' (G.R.Desiraju, T.Stainer, 1999; Grabowski S.J., 2001). Similar non-covalent interactions specify the spatial constitution of the RNA/DNA chains and their physical and chemical properties in the ground and excited states. Besides the experimental study, the NA bases are favorite objects for investigation of electron structure of the individual base molecules as well as their interactions between them (M. Preuss, et al., 2004; S. S. Wesolowski et al., 2001; Daniele Varsano et al., 2006). Aromatic $\pi-\pi$ stacking interactions are generally defined as the attractive interactions that occur between the $\pi$-clouds of aromatic systems in a parallel, face-to-face orientation. They play a fundamental role in many aspects of chemistry and biochemistry (C. A. Hunter, J. K. M. Sanders, 1991; S. K. Burley, G. A. Petsko, 1985), for example in the fields molecular recognition (E. A. Meyer et all, 2003) self-assembly (C. G. Claessens, J. F. Stoddart, 1997) supramolecular chemistry, and general host-guest interactions (B. Askew et al., 1989; D. B. Smithrud, F. Diederich, 1990; C. A. Hunter, 1994; J. Rebek, 1996). $\pi$ - $\pi$-stacking in biology is often integral to the structure and function of proteins, cofactors and substrates (G.B. McGaughey et al.,1998).

While individually weak, the additive power of these interactions leads to large effects, DNA structure being the quintessential example (J. D.Watson, H. C. D. Crick, 1953). In such intricate scenarios, very often the $\pi-\pi$ interaction is considered as some sort of "deus ex machine", intervening in reactions, stabilizing complexes, and influencing structure. Therefore, being able to estimate the energetic and structural features of these interactions would be extremely useful in modeling and understanding many important phenomena (Mark P Waller et al., 2006). The results of numerous studies show that many properties of NA bases, in particular, their affinity to other biomolecules depend on relative positions of their molecular levels, firstly, the frontier levels and levels of lone electron pairs of the two-coordinated nitrogen atoms capable to generate the hydrogen bonds. The MO energies can be estimated experimentally, for example, by photoelectron spectra (Timo Fleig, Stefan Knecht, 2007) or by quantum-chemical calculations (Vasily A. Ovchinnikov, Dage Sundholm, 2014; O.O. Brovarets' et al., 2014; Daniel Svozil et al., 2004). In addition, to obtain reasonable accuracy for small electron affinities, electronic energies have to be calculated with as high precision as possible. This criterion includes 
sustaining the accuracy in calculating the atomic integrals, tightening the convergence criteria in the SCF and post-SCF calculations, etc. Obviously, the challenge of evaluating accurate electron affinities becomes more and more difficult as the size of the molecule or complex grows (Anil Kumar et al., 2004).

Using reversible reduction potentials, Wiley (J. R. Wiley et al., 1991) obtained electron affinities of the purine and pyrimidine bases of nucleic acids experimentally. Chen (E. C. M. Chen, E. S. D.Chen, N. Sane, 1998) calculated the EAs of A, G, C, T, and Uracil (U) using semiempirical AM1-MCCI (multiconfigurational configuration interaction). Their AM1-MCCI values were found to be in good agreement with the experimental values for all the bases (E. C. M.Chen, E. S. D.Chen, 2000). Scheidt (J. Schiedt, R. Weinkauf, D. M. Neumark, E. W. Schlag, 1998) investigated the electron binding to DNA bases $\mathrm{U}, \mathrm{T}$, and $\mathrm{C}$ in the presence of water clusters using photodetachment-photoelectron spectroscopy (PD-PES). They found positive electron affinities (EA) in the $62-86 \pm 8 \mathrm{meV}$ range for dipole-bound states of the bases. For $\mathrm{U}$ and $\mathrm{T}$, they detected one dipole-bound state, while for cytosine, they found two dipole-bound states $(85 \pm 8$ and $230 \pm 8 \mathrm{meV})$.

So, as the relative positions of the frontier levels determine the main electronic properties and reaction ability, there is important necessity to compare the MO energies with energies of the other conjugated molecules. It was earlier proposed to characterize the donor properties of the linear conjugated molecules ( $\alpha, \omega$-disubstitutedpolyenes and polemethines) by the shifting of the frontier levels caused by introducing of terminal substituents (Kachkovskiy A.D., 1997). Similar approach likely can be used to theoretical estimation of the donor/acceptor properties of NA bases and their analogues.

\section{Electron donor ability of $\pi$-electron molecules}

Earlier it was proposed (Kachkovskiy A.D., 1997) to estimate quantitative donor/acceptor properties of conjugated molecules by the relative positions of their frontier molecular levels using the following formulas:

$$
\varphi_{0}=(\varepsilon \text { LUMO- } \alpha) /(\varepsilon \text { LUMO }-\varepsilon \text { HOMO }),(1)
$$

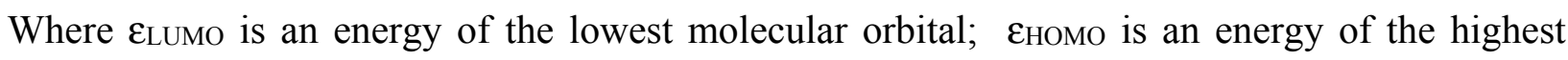
occupied MO, while $\alpha$ is so-called Coloumb integral (in framework of Hükel approximation), which corresponds to an energy of $2 p_{z}$ electron of the carbon atom in $\mathrm{sp}^{2}$-hybridyzation. In the neutral linear conjugated systems, polyenes $\mathbf{1}$, polymethine radicals $\mathbf{2}$ or polyacenes $\mathbf{3}$, as shown earlier (Pilipchuk N.V., Kachkovski O.D. et al., 2005) parameter $\alpha$ is equal the energy gap middle. 


$$
\begin{aligned}
& \mathrm{H}_{2} \mathrm{C}=\mathrm{CH}-(\mathrm{CH}=\mathrm{CH})_{n}-\mathrm{CH}=\mathrm{CH}_{2} \mathbf{1} \\
& \mathrm{H}_{2} \mathrm{C}^{\bullet}-(\mathrm{CH}=\mathrm{CH})_{n}-\mathrm{CH}=\mathrm{CH}_{2} 2
\end{aligned}
$$<smiles>CCCc1cc2cccc(CC)c2cc1CC</smiles>

Indeed, the calculations show that the frontier electron levels in chain of the unsubstitued polyenes1 approach to each other regularly upon lengthening of the conjugated chain, so that distance between them decreases, as one can see $\mathbf{3}$ demonstrably for the levels of polyenes1obtained by three various methods (M. J. Frisch, G. W. Trucks et al., 2003) in Table $1(a-c)$.

Table 1a

Unsubstitued polyenes 1.Frontier Level Energies (E, a.u.) in Hartree-Fock approximation (HF)

\begin{tabular}{|c|c|c|c|c|c|}
\hline \multirow{2}{*}{$\mathbf{n}$} & \multicolumn{5}{|c|}{ Hartree-Fock/6-31 (d,p) } \\
\cline { 2 - 6 } & HOMO & LUMO & $\boldsymbol{\Delta}^{*}$ & HOMO+ $\mathbf{\Delta / 2}$ & $\boldsymbol{\varphi}_{\mathbf{0}}$ \\
\hline $\mathbf{2}$ & $-0,27665$ & $+0,08745$ & 0,36410 & $-0,09460$ & 0,501 \\
\hline $\mathbf{3}$ & $-0,26544$ & $+0,07612$ & 0,34156 & $-0,09466$ & 0,500 \\
\hline $\mathbf{4}$ & $-0,25763$ & $+0,06822$ & 0,32585 & $-0,09471$ & 0,500 \\
\hline $\mathbf{5}$ & $-0,25196$ & $+0,06250$ & 0,31446 & $-0,09473$ & 0,500 \\
\hline $\mathbf{6}$ & $-0,24774$ & $+0,05824$ & 0,30598 & $-0,09475$ & 0,500 \\
\hline $\mathbf{7}$ & $-0,24451$ & $+0,05496$ & 0,29947 & $-0,09478$ & 0,500 \\
\hline $\boldsymbol{8}$ & $-0,24198$ & $+0,05240$ & 0,29438 & $-0,09479$ & 0,500 \\
\hline $\boldsymbol{9}$ & $-0,23990$ & $+0,05027$ & 0,29017 & $-0,09482$ & 0,500 \\
\hline $\mathbf{1 0}$ & $-0,23896$ & $+0,04861$ & 0,28757 & $-0,09518$ & 0,499 \\
\hline
\end{tabular}

Table $1 b$

Unsubstituted polyenes 1. Frontier Level Energies ((E, a.u.) in DFT/CAM-B3LYP

\begin{tabular}{|c|c|c|c|c|c|}
\hline \multirow{2}{*}{$\mathbf{n}$} & \multicolumn{5}{|c|}{ DFT/CAM-B3LYP } \\
\cline { 2 - 6 } & HOMO & LUMO & $\boldsymbol{\Delta}$ & HOMO+ $\mathbf{\Delta} / \mathbf{2}$ & $\boldsymbol{\varphi}_{\mathbf{0}}$ \\
\hline $\mathbf{2}$ & $-0,24680$ & $-0,01062$ & 0,23618 & $-0,12871$ & 0,503 \\
\hline $\mathbf{3}$ & $-0,23694$ & $-0,02094$ & 0,21600 & $-0,12894$ & 0,502 \\
\hline $\mathbf{4}$ & $-0,22990$ & $-0,02830$ & 0,20160 & $-0,12910$ & 0,502 \\
\hline $\mathbf{5}$ & $-0,22465$ & $-0,03375$ & 0,19090 & $-0,12920$ & 0,501 \\
\hline $\mathbf{6}$ & $-0,22063$ & $-0,03794$ & 0,18269 & $-0,12929$ & 0,501 \\
\hline $\mathbf{7}$ & $-0,21746$ & $-0,04119$ & 0,17627 & $-0,12933$ & 0,501 \\
\hline $\mathbf{8}$ & $-0,21494$ & $-0,04383$ & 0,17111 & $-0,12939$ & 0,500 \\
\hline $\mathbf{9}$ & $-0,21288$ & $-0,04592$ & 0,16696 & $-0,12940$ & 0,500 \\
\hline $\mathbf{1 0}$ & $-0,21120$ & $-0,04766$ & 0,16354 & $-0,12943$ & 0,500 \\
\hline
\end{tabular}


Table 1-c

Unsubstitued polyenes 1.Frontier Level Energies ( $\mathcal{E}_{\text {, a. a.) }}$ in DFT/wB97XD

\begin{tabular}{|c|c|c|c|c|c|}
\hline \multirow{2}{*}{$\mathbf{n}$} & \multicolumn{5}{|c|}{ DFT/wB97XD } \\
\cline { 2 - 6 } & HOMO & LUMO & $\boldsymbol{\Delta}$ & HOMO+ $\mathbf{\Delta} \mathbf{2}$ & $\boldsymbol{\varphi}_{\mathbf{0}}$ \\
\hline $\mathbf{2}$ & $-0,26760$ & $+0,00837$ & 0,27597 & $-0,12962$ & 0,504 \\
\hline $\mathbf{3}$ & $-0,25764$ & $-0,00220$ & 0,25544 & $-0,12992$ & 0,503 \\
\hline $\mathbf{4}$ & $-0,25047$ & $-0,00977$ & 0,2407 & $-0,13012$ & 0,503 \\
\hline $\mathbf{5}$ & $-0,24517$ & $-0,01556$ & 0,22961 & $-0,13037$ & 0,502 \\
\hline $\mathbf{6}$ & $-0,24104$ & $-0,01972$ & 0,22132 & $-0,13038$ & 0,502 \\
\hline $\mathbf{7}$ & $-0,23792$ & $-0,02326$ & 0,21466 & $-0,13059$ & 0,501 \\
\hline $\boldsymbol{8}$ & $-0,23543$ & $-0,02592$ & 0,20951 & $-0,13068$ & 0,501 \\
\hline $\mathbf{9}$ & $-0,23339$ & $-0,02813$ & 0,20526 & $-0,13076$ & 0,500 \\
\hline $\mathbf{1 0}$ & $-0,23171$ & $-0,02990$ & 0,20181 & $-0,13081$ & 0,500 \\
\hline
\end{tabular}

However, the energy gap, $\delta / 2=\varepsilon$ номо $+\left(\varepsilon_{\text {LUMO }}+\varepsilon_{\text {Hомо }}\right) / 2$, remains practically same independently on the chain length $\mathrm{n}$. Although, the DFT methods taking into consideration the electron correlation give the regular somewhat increasing of $\mathrm{MO}$ energies because of the considerable polarization of the $\mathrm{C}-\mathrm{H}$ bonds. In the Huekel method, the energy gap in the unsubstituted polyenes 1 correspond to Coulomb integral $\alpha$ by the definition, as can be seen from Figure 1.

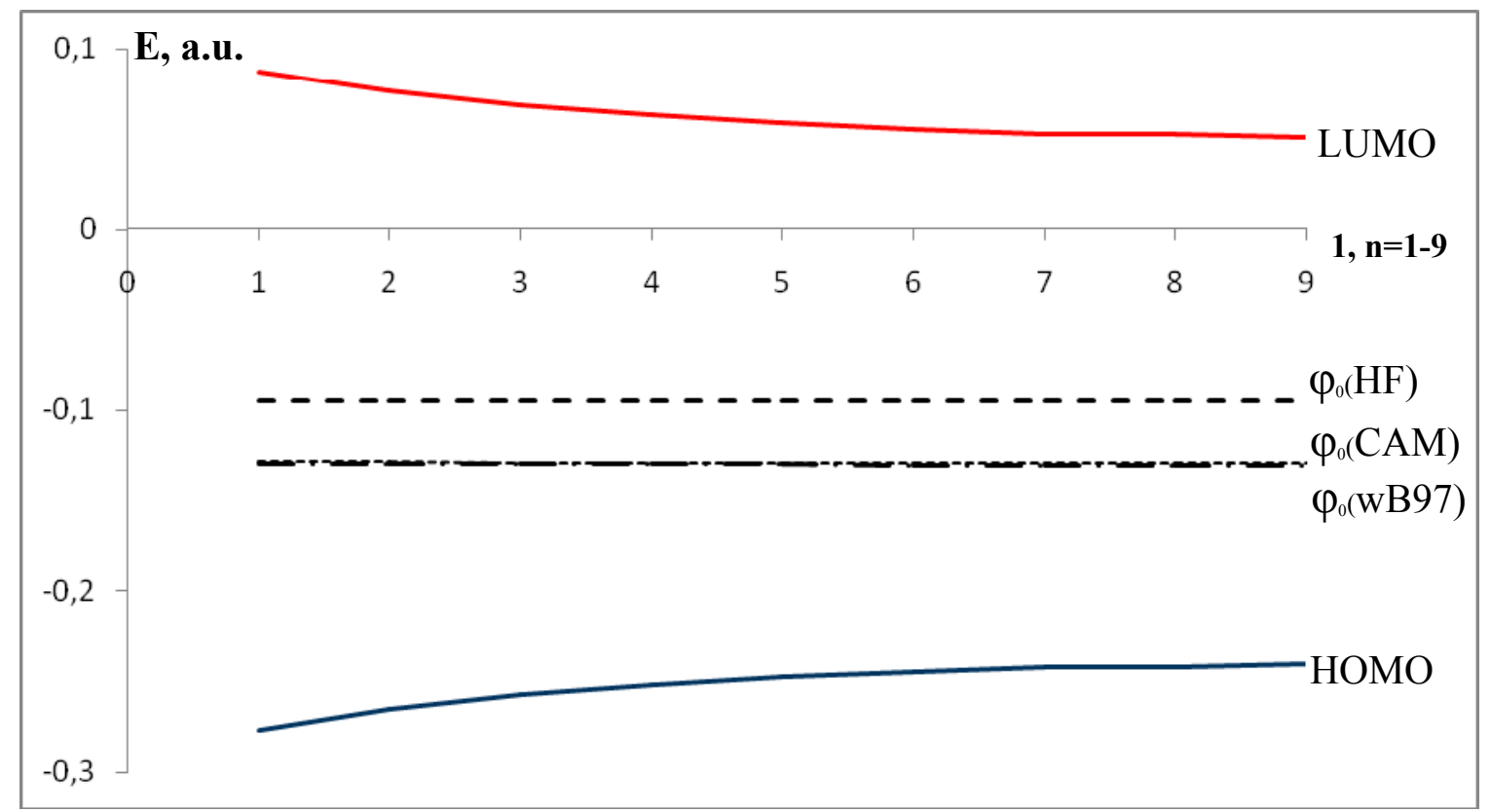

Figure 1. Energies of frontier MOs and energy gap middle, $\delta / 2=\varepsilon_{\mathrm{HOMO}}+\left(\varepsilon_{\mathrm{LUMO}}+\varepsilon_{\mathrm{HOMO}}\right) / 2$, inpolyenes1 calculated by HF/6-31 (d,p); DFT/CAM-B3LYPandDFT/wB97XD methods 
Then, we could treate that:

$$
\alpha=\delta / 2=\varepsilon \text { HOMO }+(\varepsilon \text { LUMO }-\varepsilon \text { HOMO }) / 2 \text { upon } \mathrm{n} \rightarrow \operatorname{maximum}(2)
$$

Similarly, the frontier levels energies for the $\alpha$ - and $\beta$-electrons in the polymethine radicals $\mathbf{2}$ demonstrate the analogical dependence on chain length n, what is seen in Figure 2.

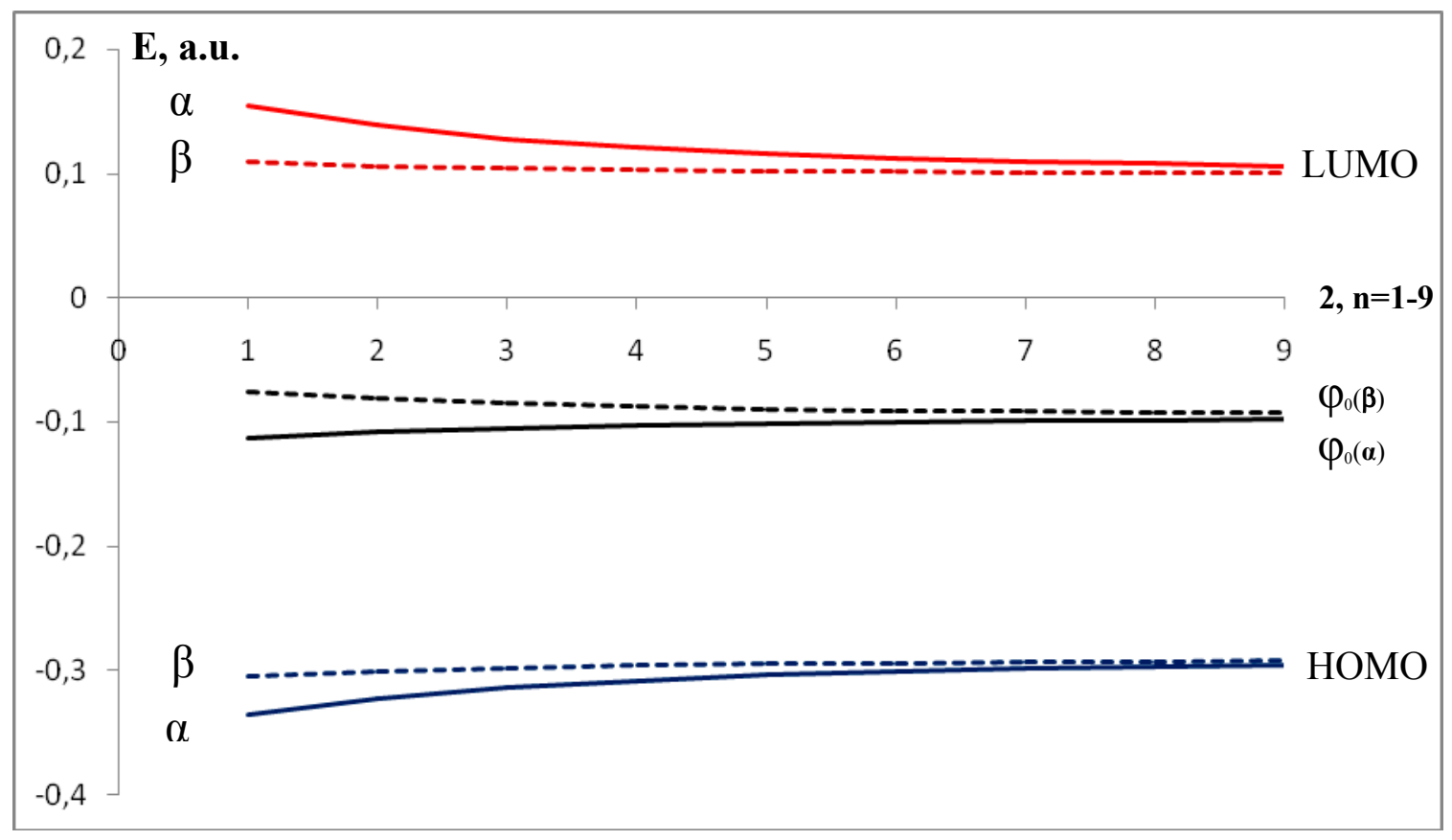

Figure 2. Level energies and energy gap middle for $\alpha$-electrons (-) and $\beta$-electrons (----) in neutral polymethine radicals $\mathbf{2}(\mathbf{H F})$.

It is clear seen that the levels for electron with the opposite spins approach regularly; the gap middles approach also, so that the value $\delta / 2$ tends to the magnitude-0.095a.u., found for the polyenes $\mathbf{1}$.

The practically the same value $\delta / 2$ is obtained for the third type of neutral linear conjugated molecules 3; the positions of both frontier levels as well as the position of the gap middle are presented in Figure 3. 


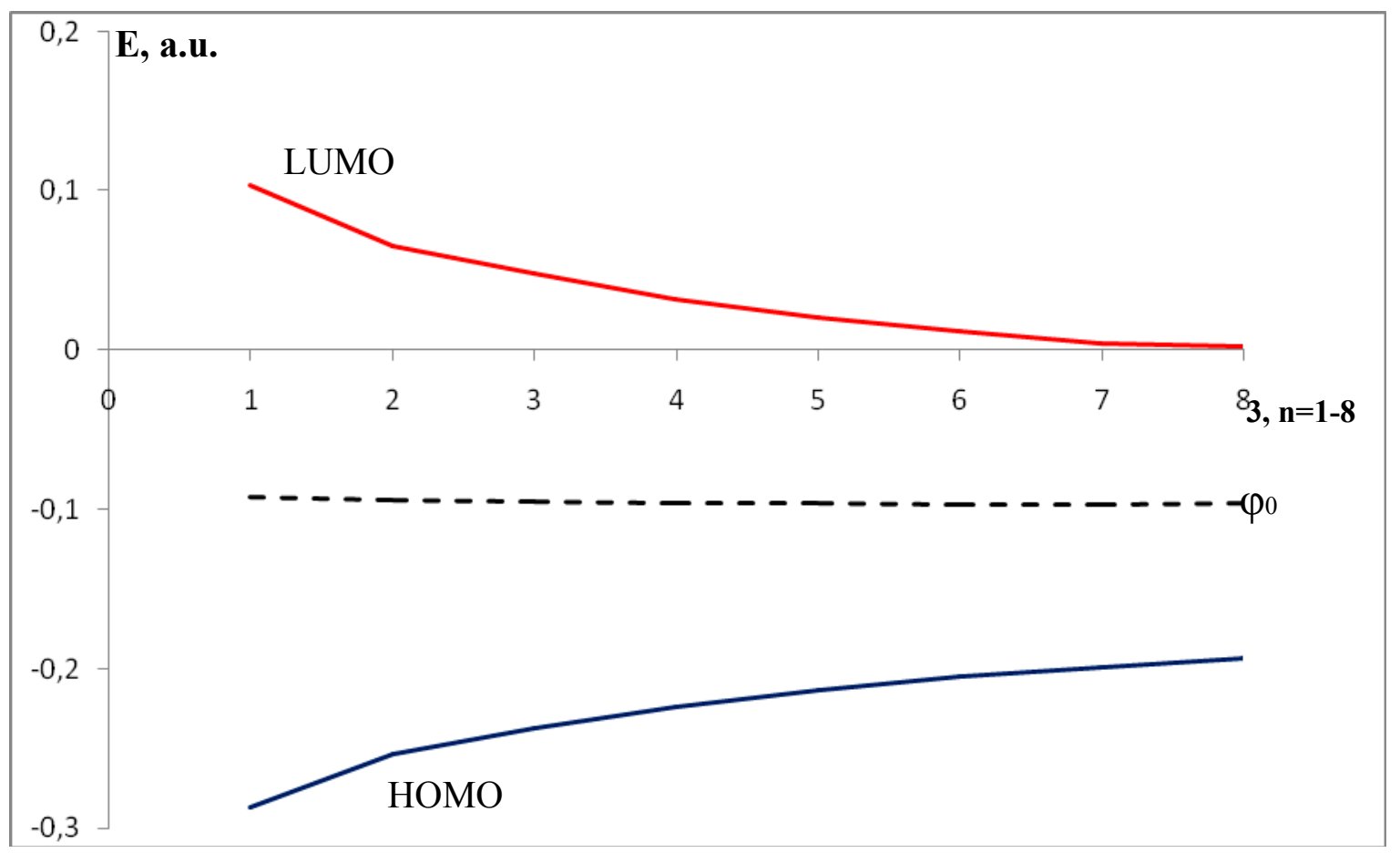

Figure 3. Energies of frontier MOs as well as energy gap middle, $\delta / 2$, inacenes 3

Thus, the energy gap middle is established to be practically the same and hence enables determine the Coulomb integral, $\alpha$, in formulas (1). For the three examples 1, 2, 3, the parameter $\varphi_{0}=1 / 2$ for the enough long conjugated molecules.

On the other hand, in the examples above, the donor and acceptor properties are balance and consequently could be considered as conjugated molecules where the frontier levels are disposed symmetrically relative to the centra inreference electron levels. Then, shifting of frontier levels (upon the same energy gap) down can be treated as decreasing of a donor ability or/and increasing of an acceptor ability. I.e., the parameter $\varphi_{0}$ can be used for the quantitative estimation of a donor/acceptor strength or a donor/acceptor ability. By definition, the $\varphi_{0}=1 / 2$ means that the conjugated system is balanced, as regards to its donor/acceptor properties. According to the formulas (1), increasing of the value $\varphi_{0}$ points directly on increasing of the acceptor ability; the proposed parameter of relative donor/acceptor ability of conjugated system with stable electron shell lies within the range: $0 \leq \varphi_{0} \leq 1$. If value $\varphi_{0}>0.5$, then molecule is acceptor, while $\varphi_{0}<0.5$ indicates on the prevalent donor property. If value $\varphi_{0}>0.5$, then molecule is a donor, as it visually pictured in Figure 4b: the frontier levels are shifted up in compare the energy gap of the reference molecule (for example, polyene with the same energy gap) presented in Fig. 4a. While $\varphi_{0}<0.5$, then it indicates on the prevalent donor property of the conjugated molecule, what is illustrated by Figure 4(c). 


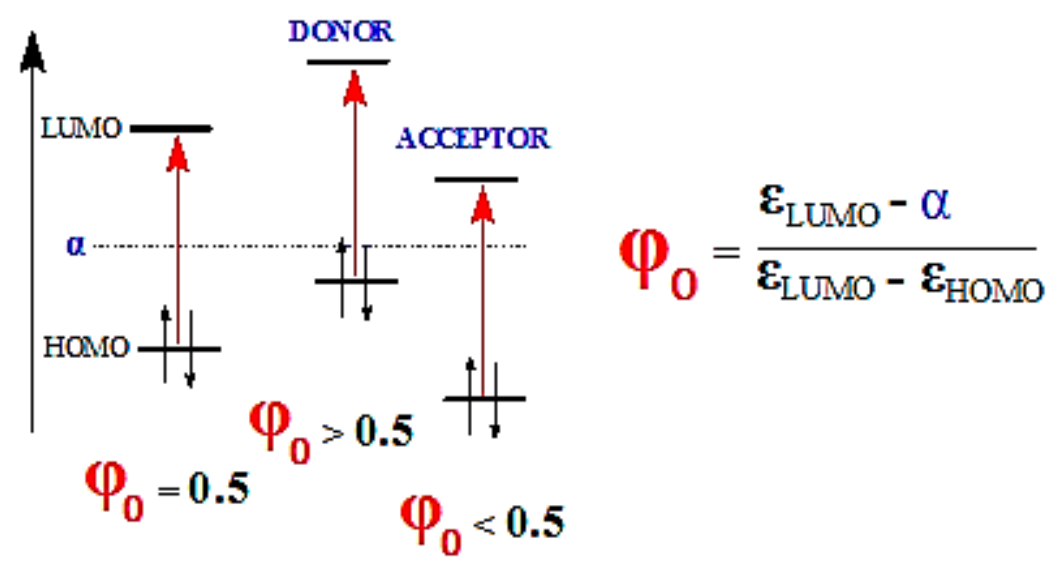

a b c

Figure 4. Relative energy gap position in reference molecule (a), donor molecule (b) and acceptor molecule (c).

In the study of the polymethine dyes and $\alpha, \omega$-disubstitued polyenes, the parameter $\varphi_{0}$ point on the shifting the energy gap upon introducing of the donor/acceptor terminal residues. The analogical approach could be used for estimation the donor/acceptor conjugated molecules, particularly, various heterocycles, including the DNA bases.

\section{Donor abilities of heterocycles}

For sake an illustration, let us present the simplest heterocycles containing the nitrogen atom and/or other heteroatoms. In Table 2, the frontier level energies as well as the parameter $\varphi_{0}$ for the 6-membered nitrogen-containing heterocycles 4-6 and 5-membered heterocycle 7-8 are collected.<smiles>c1ccncc1</smiles>

4<smiles>c1cncnc1</smiles>

5<smiles>c1ncncn1</smiles>

6<smiles>[X]c1ccccc1</smiles>

7<smiles></smiles>

8

Table 2

Frontier level energies (E, a.u.) of heterocyclic compounds 4-8(HF)

\begin{tabular}{|c|c|c|c|c|c|c|}
\hline Molecule & $\mathbf{X}$ & HOMO & LUMO & $\Delta^{*}$ & $\delta / 2$ & $\varphi_{0}$ \\
\hline $\mathbf{4}$ & & $-0,34403$ & $+0,12661$ & 0,47064 & $-0,10871$ & $\mathbf{0 , 4 7 1}$ \\
\hline $\mathbf{5}$ & & $-0,37681$ & $+0,11098$ & 0,48779 & $-0,13292$ & $\mathbf{0 , 4 2 3}$ \\
\hline $\mathbf{6}$ & & $-0,43684$ & $+0,10198$ & 0,53882 & $-0,16743$ & $\mathbf{0 , 3 6 6}$ \\
\hline $\mathbf{7}$ & $\mathbf{N}-\mathbf{H}$ & $-0,29270$ & $+0,20678$ & 0,49948 & $-0,04296$ & $\mathbf{0 , 6 0 4}$ \\
\hline & $\mathbf{O}$ & $-0,31832$ & $+0,17848$ & 0,4968 & $-0,06992$ & $\mathbf{0 , 5 5 1}$ \\
\hline
\end{tabular}




\begin{tabular}{|c|c|c|c|c|c|c|}
\hline & $\mathbf{S}$ & $-0,32859$ & $+0,13707$ & 0,46566 & $-0,09576$ & $\mathbf{0 , 4 9 9}$ \\
\hline $\mathbf{8}$ & $\mathbf{N}-\mathbf{H}$ & $-0,31928$ & $+0,19522$ & 0,51450 & $-0,06203$ & $\mathbf{0 , 5 6 4}$ \\
\hline & $\mathbf{O}$ & $-0,35032$ & $+0,16558$ & 0,51590 & $-0,09237$ & $\mathbf{0 , 5 0 5}$ \\
\hline & $\mathbf{S}$ & $-0,34771$ & $+0,12310$ & 0,47081 & $-0,11231$ & $\mathbf{0 , 4 6 4}$ \\
\hline $\mathbf{1 , n}=10$ & $-0,23896$ & $+0,04861$ & 0,28757 & $-0,09518$ & $\mathbf{0 , 5}$ \\
\hline
\end{tabular}

The 6-membered nitrogen cycles 4-6 should be treated as electron-balanced $\pi$-systems: a number of $\pi$-electrons is equal to a number of $\pi$-centers. One can see from Table 2 that introducing of the nitrogen atoms in benzene cycle ( $\pi$-electron-balanced molecule) is accompanied by regular shifting of the frontier level positions down. Then parameter $\varphi_{0}$ decreases regularly every time upon replacement of $\mathrm{CH}$ group by another nitrogen atom: $\varphi_{0}=0,471$ (pyridine), 0.423 (pyrimidine), 0.366 (triazine), i.e. the energy gap shifts regularly down. Consequently, it indicates increasing of an acceptor ability, and/or decreasing of a donor ability.

In a contrast, the 5-membered cycles 7 should be considered as electron-sufficient conjugated molecules: a number of $\pi$-electrons exceed a number of $\pi$-centers. Then both frontier levels shift thereafter up so that the gap middle is found closer to the highest occupied level; in accordance with this, the parameter $\varphi_{0}$ increasesalsowhat point in increasing of the donor ability. The variation of the heteroatom $\mathrm{X}$ cause change of the donor/acceptor properties upon going from furan $(\mathrm{X}=\mathrm{O})$ to azole $\left(\mathrm{X}=\mathrm{NCH}_{3}\right)$ or thiophene $(\mathrm{X}=\mathrm{S}): \varphi_{0}=0.604 ; 0.551 ; 0.499$, i.e. the decreasing of the heteroatom $\mathbf{X}$ electrononegativity is accompanied by regular decreasing of the acceptor ability.

Introducing additionally of the nitrogen atom in the 5-membered heterocycle $\mathbf{8}$ causes the shifting of energy gap down, and hence such chemical change increases acceptor ability, so that $\delta \varphi_{0} \approx 0.6$ upon going from $\mathbf{8}, \mathrm{X}=\mathrm{NH}$ to $\mathbf{8}, \mathrm{X}=\mathrm{O}$ and $\delta \varphi_{0} \approx 0.4$ upon going from $\mathbf{8}, \mathrm{X}=\mathrm{O}$ to $\mathbf{8}, \mathrm{X}$ $=\mathrm{S}$.

It is to be also noted that the HOMO and the LUMO demonstrate the different sensitivities to the changes of the chemical constitution and hence the change of the energy gap $\Delta$ does not correlate with the donor/acceptor parameter $\varphi_{0}$.

\section{Estimation of donor/acceptor ability of NA bases}

Generally, the bases in the DNA subunits are connected sequentially with ribose, deoxyribose and phosphorus residues. However here we will study the model heterocylic bases 9-13, when the base tails are modeled by the saturated methyl $\left(-\mathrm{CH}_{3}\right)$ group (Figure 4). 
<smiles>Cn1cnc2c(N)ncnc21</smiles>

9 (A)<smiles>Cn1cnc2c(=O)[nH]c(N)nc21</smiles>

$10(\mathbf{G})$<smiles>Cn1ccc(=O)[nH]c1=O</smiles>

$11(\mathrm{U})$<smiles>Cn1ccc(N)nc1=O</smiles>

$12(\mathrm{C})$<smiles>Cc1cn(C)c(=O)[nH]c1=O</smiles>

$13(\mathrm{~T})$

Figure 4. General structure of studied purine and pyrimidine bases.

The calculated frontier level energies and other characteristics of the studied purine and pyrimidine bases 9-13 are collected in Table 3 .

Table 3

Frontier level energies $\left(\mathcal{E}_{i}\right.$, a.u.) of purine $(\mathbf{9}, 10)$ and pyrimidine $(11-13)$ bases

\begin{tabular}{|c|c|c|c|c|c|c|}
\hline Molecule & Method & HOMO & LUMO & $\Delta$ & $\delta / 2$ & $\varphi_{0}$ \\
\hline \multirow[t]{3}{*}{$\mathbf{9}, \mathbf{A}$} & HF & $-0,30354$ & $+0,13958$ & 0,44312 & $-0,08198$ & 0,530 \\
\hline & DFT/CAM & $-0,26523$ & $+0,03594$ & 0,30117 & $-0,06622$ & 0,549 \\
\hline & DFT/wB & $-0,28686$ & $+0,05595$ & 0,34281 & $-0,09853$ & 0,545 \\
\hline \multirow{3}{*}{$10, G$} & HF & $-0,28987$ & $+0,15744$ & 0,44731 & $-0,09904$ & 0,565 \\
\hline & DFT/CAM & $-0,25069$ & $+0,05364$ & 0,30433 & $-0,11883$ & 0,602 \\
\hline & DFT/wB & $-0,27245$ & $+0,07438$ & 0,34683 & $-0,14236$ & 0,592 \\
\hline \multirow[t]{3}{*}{$11, \mathrm{U}$} & HF & $-0,35216$ & $+0,11451$ & 0,46667 & $-0,14289$ & 0,449 \\
\hline & DFT/CAM & $-0,29745$ & $+0,01274$ & 0,31019 & $-0,10164$ & 0,458 \\
\hline & DFT/wB & $-0,31888$ & $+0,03311$ & 0,35199 & $-0,1243$ & 0,466 \\
\hline \multirow{3}{*}{$12, \mathrm{C}$} & HF & $-0,32835$ & $+0,12507$ & 0,45342 & $-0,1248$ & 0,486 \\
\hline & DFT/CAM & $-0,27500$ & $+0,02641$ & 0,30141 & $-0,09542$ & $\mathbf{0 , 5 1 7}$ \\
\hline & DFT/wB & $-0,29654$ & $+0,04695$ & 0,34349 & $-0,1186$ & 0,517 \\
\hline \multirow[t]{3}{*}{$13, T$} & HF & $-0,31833$ & $+0,12749$ & 0,44582 & $-0,11922$ & 0,499 \\
\hline & DFT/CAM & $-0,2664$ & $+0,02920$ & 0,2956 & $-0,11546$ & $\mathbf{0 , 5 3 7}$ \\
\hline & DFT/wB & $-0,28818$ & $+0,04975$ & 0,33793 & $-0,06622$ & 0,534 \\
\hline \multirow[t]{3}{*}{$1, n=10$} & HF & $-0,23896$ & $+0,04861$ & 0,28757 & $-0,09518$ & 0,5 \\
\hline & DFT/CAM & $-0,21120$ & $-0,04766$ & 0,16354 & $-0,12943$ & 0,5 \\
\hline & DFT/wB & $-0,23171$ & $-0,02990$ & 0,20181 & $-0,13081$ & 0,5 \\
\hline
\end{tabular}

At first, all three used methods give the comparatively close values for the proposed donor/acceptor parameter $\varphi_{0}$, in spite of the more essential differences in MO energies. The both DFT-method staking into consideration the electron correlation give somewhat higher values than simple HF approximation with the bases set. However, all three methods show the same 
sensitivities of donor/acceptor parameter on the chemical constitution of the DNA/RNA heterocyclic bases.

So, three bases, $\mathbf{U}, \mathbf{C}, \mathbf{T}$, are composed of 6-memvered cycle with the exocyclic onecoordinated oxygen atom giving one $2 \mathrm{p}_{z}$ electron in total conjugated system; also, cyctosine and thymine have the exocyclic amino group with two electrons included in the $\pi$-system, so that each molecule has its stable electron shell. Because of the heavy atoms, the heterocyclic bases $\mathbf{C}$ and $\mathbf{T}$ should demonstrate the principally acceptor properties. As regards to $\mathbf{U}$, it contains two $t$ one-coordinated oxygen atoms (giving two electrons in the total $\pi$-system); two tri-coordinated nitrogen atoms give four electrons (at the expense of their LEPs). But, though bases $\mathbf{U}, \mathbf{C}, \mathbf{T}$ are electron-sufficient systems, so they are acceptors because of the heavy nitrogen atoms existence in their structure.

In contrast, the heterocyclic bases $\mathbf{A}$ and $\mathbf{G}$ contains 5-membered cycle with the tricoordinated nitrogen atom with its LEP with makes these molecules as high donor $\pi$-system; thus, their parameters are higher the middle value: $\varphi_{o}>0.5$, i.e. they should give evidence of their donor properties. The tendency predicted all used methods is seen from presented data in Table 3 to be the same.

In simple approaches of molecular orbital theory, the HOMO energy ( $\left.\varepsilon_{\mathrm{HOMO}}\right)$ is related to the ionization potential (IP) by Koopmanns' theorem and the LUMO energy ( $\varepsilon$ LUMO) has been used to estimate the electronaffinity (EA). As it seen from the Table 4, parameter $\varphi$ changing in the same direction as measured practically ionization potential (Orlov V. M., Smirnov A. N., Varshavsky Ya. M., 1976) and electronaffinity (J. R.Wiley, J. M. Robinson et al., 1991).

Table 4

Correlation of the calculated parameter $\boldsymbol{\varphi}$ with practically measured EA and IP.

\begin{tabular}{|c|c|c|c|c|}
\hline Bases & $\boldsymbol{\varphi}$ & $\mathbf{E A}, \mathbf{e V}$ & $\mathbf{1 -} \boldsymbol{\varphi}$ & $\mathbf{I P}, \mathbf{e V}$ \\
\hline $\mathbf{1 0}(\mathbf{G})$ & 0.566 & 1.51 & 0.434 & 7.77 \\
\hline $\mathbf{9}(\mathbf{A})$ & 0.530 & 0.95 & 0.470 & 8.26 \\
\hline $\mathbf{1 2}(\mathbf{C})$ & 0.472 & 0.56 & 0.528 & 8.68 \\
\hline $\mathbf{1 3}(\mathbf{T})$ & 0.445 & 0.79 & 0.555 & 8.87 \\
\hline $\mathbf{1 1}(\mathbf{U})$ & 0.431 & 0.80 & 0.569 & 9.32 \\
\hline
\end{tabular}

Nevertheless our experimental data confirm the general conclusion made of these publications, that the purine bases are better electron donors than the pyrimidines.

\section{Comparison of donor/acceptor abilities of the heterocyclic bases of NA}

It is known that the RNA/DNA bases make up the bases pair in DNA chain molecules, so that thestable A-T and G-C pairs are formed, they are connecting by two/three bonding bonds 
(Zinger, 1984). Besides, the pyrimidines are bonded with the purines; other possible pairs turn out to be unstable. Then, let us compare the indicesof the pyrimidine bases with the reference long polyene molecule $\mathbf{1}$ and the pyrimidine 5. The Figure 5 shows distinctly that the $\mathbf{U}$ (uracil) and $\mathbf{C}$ (cytosine) should predominately treat as acceptors, similarly to the pyrimidine heterocycle 5, as far as their $\varphi_{0}<0.5$. Introducing of the donor methyl $\left(-\mathrm{CH}_{3}\right)$ substituent in the uracil and hence going to thymin (T) leads to shifting of the frontier levels up and to decreasing of the acceptor property (see increasing of the index $\varphi_{0}$ in Table 3).

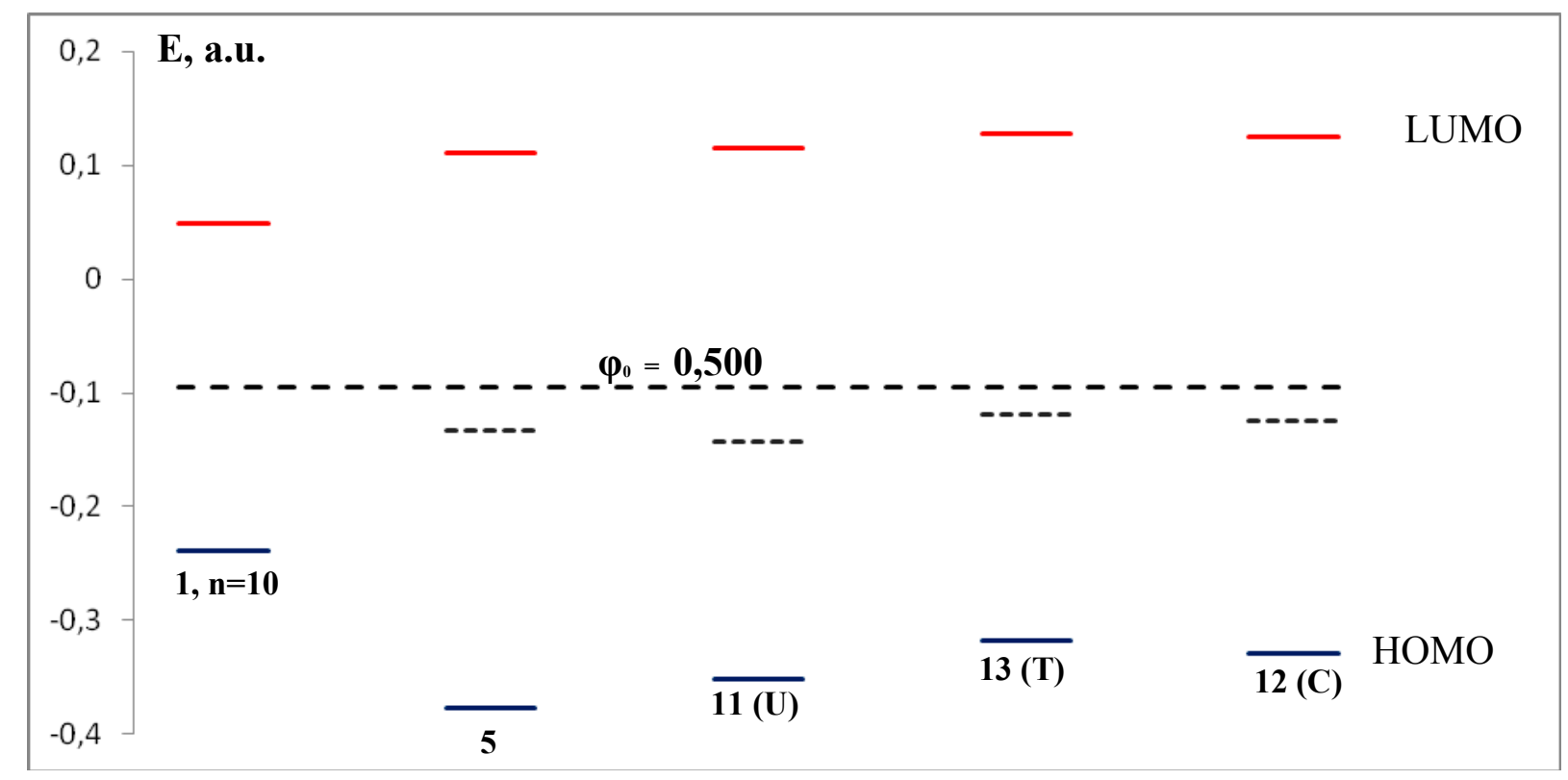

Figure 5. Relative positions of pyrimidine bases $\mathbf{U}, \mathbf{T}, \mathbf{C}$ and reference compound

$$
1 \text { and } \mathbf{5}\left(--\varphi_{0}\right)
$$

Inversely, the energy gap of both purine bases, $\mathbf{A}$ and $\mathbf{G}$, containing the electronexcessive 5-membered cycle, is shifted up relatively to the reference molecules $\mathbf{1}, \mathrm{n}=10$; such relative disposition of the frontier levels resembles the high donor imidazaole7, but is opposite to the acceptor pyrimidine, what is visually seen in Figure 6. 


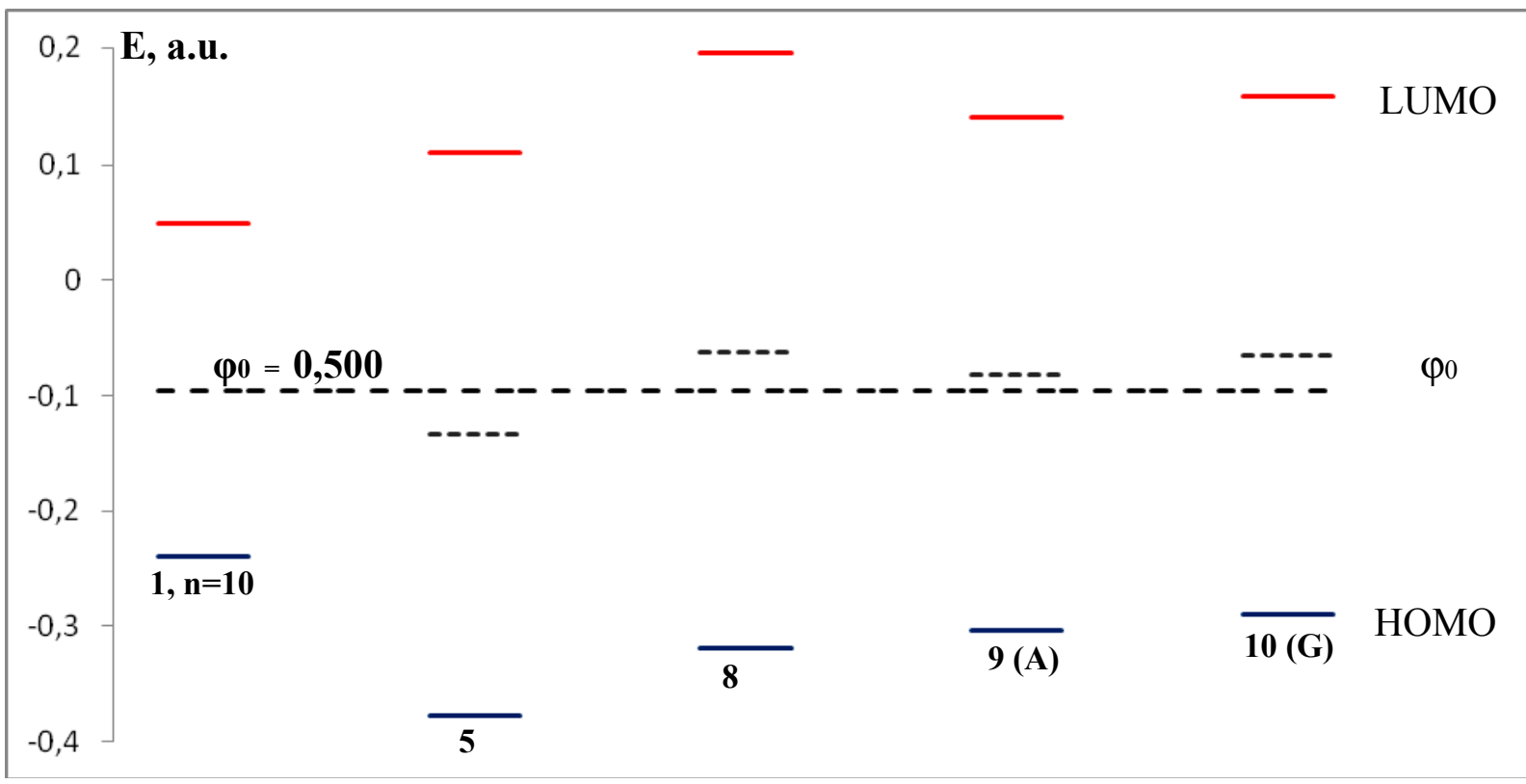

Figure 6. Relative positions of purine bases $\mathbf{A}$ and $\mathbf{G}$ and reference compound 1,5, 8 (- - - $\left.\varphi_{0}\right)$.

We could assume that donor/acceptor properties (and hence index $\varphi_{0}$ ) influence on the charge distribution and hydrogen bonding strength. It is interested to compare the index of each RNA/DNA base as well as compare the difference of the indices the pair of the corresponding bases connected by the hydrogen bonding. The calculated indices are collected in Figure 7.

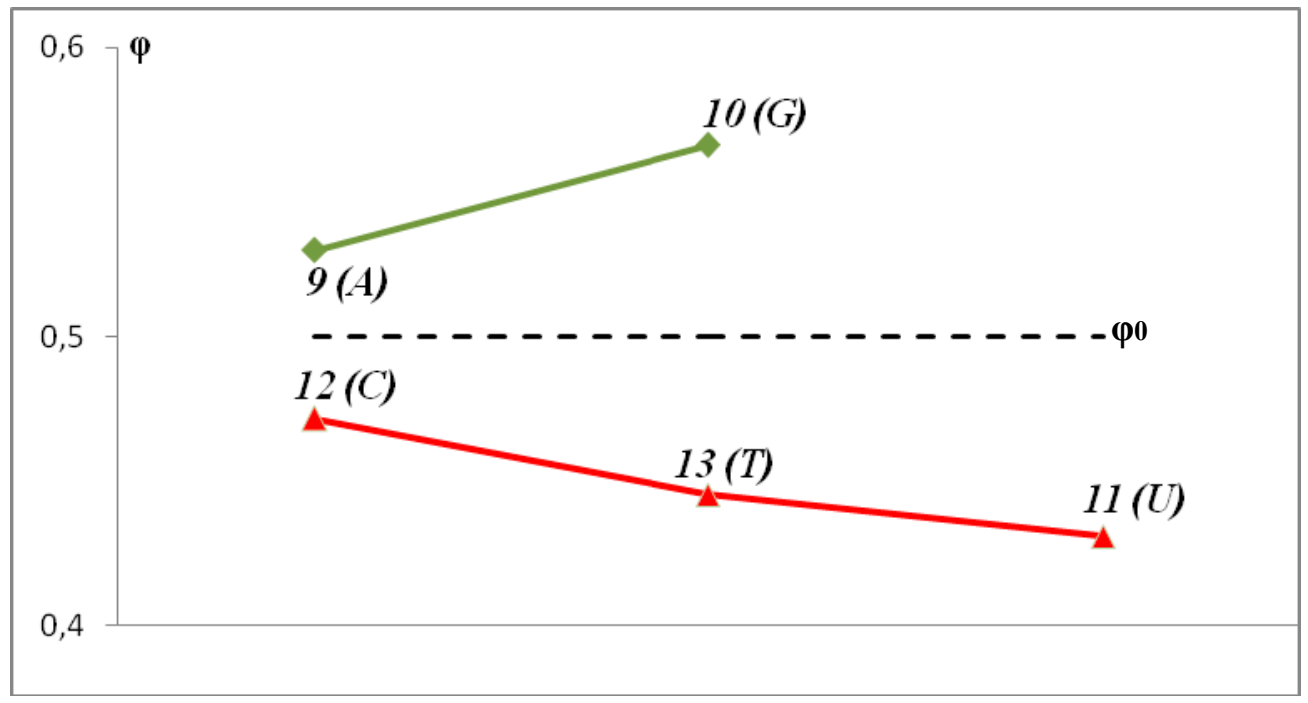

Figure 7. Indices $\varphi_{0}$ for RNA/DNA bases

One can see that the bonding bases pairs are formed so that the index $\boldsymbol{\varphi}_{0}>\boldsymbol{0 . 5}$ for the first base while $\varphi_{0}<\mathbf{0 . 5}$ for the second base, whereas other possible pairs are less stable. It is to be notablypointed that the base pairs with maximum or minimum difference of indices $\Delta \varphi_{0}$ are 
likely not stable. Evidently, there is certain optimal difference $\Delta \varphi_{0}$ in order to the base pair should be stable and also enables the DNA replication. The existence of the optimal difference in donor/acceptor properties seemly explains the well-known complementarity rule of nucleobases (Zinger, 1984).

\section{Conclusion}

Thus, an analysis of the relative position of the frontier levels (calibrated by the energy gap) enables the investigation of the donor/acceptor properties of the RNA/DNA bases more detailed. The index $\varphi_{0}$ is proposed for the quantitative quantum-chemical estimation of the donor ability of the conjugated molecules: it points on the shifting of the energy gap relative to the reference electron balanced system. The RNA/DNA bases divided strictly by two groups: a) predominantly donors $\left(\varphi_{0}>0.5\right)$; b) predominantly acceptors $\left(\varphi_{0}>0.5\right)$. Each representative base of the first group forms the stable base pair the representative base of the second group (complementarity rule) the difference of indices $\Delta \varphi_{0}$ should be optimal to enables the DNA replication. 


\section{Literature}

Zinger.(1984). Principles of Nucleic Acid Structure.Springer-Verlag.New-York-BerlinHeldelberg-Tokyo. -584.

G.R.Desiraju, T.Stainer. (1999). The weak hydrogen bond on structural chemistry and biology. New York, Oxword University Press Inc. - 526.DOI: 10.1021/ja0047368

Grabowski S.J. (2001). Hydrogen bonding strength. - Measures based on geometric and topological parameters.Journal of Physical Organic ChemistryA.-105. - 1073910746.DOI: $10.1002 /$ poc. 685

M. Preuss, W. G. Schmidt, K. Seino, J. Furthmüller, F. Bechstedt. (2004). Ground- and ExcitedState Properties of DNA Base Molecules from Plane-Wave Calculations Using Ultrasoft Pseudopotentials. Journal ofComputationalChemistry - 25. -112-122.DOI: 10.1002/jcc.10372

Steven S. Wesolowski, Matthew L. Leininger, Plamen N. Pentchev,Henry F. Schaefer. (2001). ElectronAffinitiesofthe DNA and RNA Bases.Journal ofAmericanChemicalSociety- 123.-40234028.DOI: $10.1021 / \mathrm{ja} 0038140$

DanieleVarsano, RosaDiFelice, Miguel A. L. Marques, AngelRubio. (2006). A TDDFT study of the excited states of DNA bases and their assemblies.TheJournal of Physical Chemistry. B. -110. - 7129-7138.DOI: $10.1021 / \mathrm{jp} 056120 \mathrm{~g}$

C. A.Hunter, J. K. M.Sanders. (1991). $\pi-\pi$ interactions: the geometry and energetics of phenylalanine-phenylalanine interactions in proteins. Journal of Molecular Biology-218(4). 837-846. DOI: 10.1016/0022-2836(91)90271-7

S. K. Burley, G. A. Petsko. (1985). Aromatic-aromatic interaction: a mechanism of protein structure stabilization.Science.- 229. -23-28.

E. A. Meyer, R. K.Castellano, F. AngewDiederich. (2003). Interactions with Aromatic Rings in Chemical and Biological Recognition. Angewandte Chemie International Edition.-42. -12101250.DOI: 10.1002 /anie.200390319

C. G. Claessens, J. F.Stoddart. (1997). Review.Commentary $\pi-\pi$ interactions in selfassembly.Journal of Physical Organic Chemistry-10.-254-272.DOI: 10.1002/(SICI)10991395(199705)10:5<254::AID-POC875>3.0.CO;2-3

B. Askew, P. Ballester, C. Buhr, K. S. Jeong, S. Jones, K. Parris, K. Williams, J. Rebek. (1989). Molecular recognition with convergent functional groups. VI. Synthetic and structural studies with a model receptor for nucleic acid components. Journal ofAmerican Chemical Society-111.1082-1090.DOI: $10.1021 / \mathrm{ja} 00185 \mathrm{a} 044$

D. B. Smithrud, F. Diederich. (1990). Strength of molecular complexation of apolar solutes in water and in organic solvents is predictable by linear free energy relationships: a general model 
for solvation effects on apolar binding. Journal ofAmerican Chemical Society-112.-339343.DOI: $10.1021 / \mathrm{ja} 00157 \mathrm{a} 052$

C. A. Hunter. (1994).Meldola Lecture.The role of aromatic interactions in molecular recognition.Journal ofChemical Society Reviews-23.-101-110.DOI: 0.1039/CS9942300101

J. Rebek. (1996). Assembly and encapsulation with self-complementary molecules. Journal of Chemical Society Reviews -25.-p.255-264.

G. B. McGaughey, M. Gagne, A. K. Rappe. (1998). $\pi$-Stacking interactions. Alive and well in proteins.Journal of Biological Chemistry - 273. - 15458-15463.DOI: 10.1074/jbc.273.25.15458

J. D.Watson, H. C. D. Crick. (1953). Molecular structure of nucleic acids: a structure for deoxyribose nucleic acid. Nature.-171. -737-738.

Mark P Waller, ArturoRobertazzi, James A. Platts, David E. Hibbs, Peter A. Williams. (2006). Hybrid Density Functional Theory for $\pi$-Stacking Interactions: Application to Benzenes, Pyridines, and DNA Bases. Journal of Computational Chemistry-27.-491504.DOI: $10.1002 /$ jcc. 20363

Timo Fleig, Stefan Knecht. (2007). Quantum-Chemical Investigation of the Structures and Electronic Spectra of the Nucleic Acid Bases at the Coupled Cluster CC2 Level. Journal of Physical Chemistry,A.-111.-5482-5491.DOI: 10.1021/jp0669409

Vasily A. Ovchinnikov, Dage Sundholm. (2014). Coupled-cluster and density functional theory studies of thee lectronic 0-0 transitions of the DNA bases.Physical Chemistry Chemical Physics - 16. - 6931 - 6941.DOI: $10.1039 / \mathrm{c} 3 \mathrm{cp55080j}$

O.O. Brovarets',Ye.P. Yurenko, D.M. Hovorun (2014). Intermolecular $\mathrm{CH} \cdots \mathrm{O} / \mathrm{N} \cdot \mathrm{H}$-bonds in the biologically important pairs of natural nucleobases: a thorough quantum-chemical study. Journal of Biomolecular Structure \& Dynamics.-32(6)-993-1052. DOI: $10.1080 / 07391102.2013 .799439$

Daniel Svozil1, PavelJungwirth,ZdeněkHavlas (2004). Electron binding to nucleic acid bases. Experimental and theoretical studies. Review. Collectionof Czechoslovak Chemical Communications.- 69. -1395-1428.DOI: $10.1135 / \operatorname{cccc} 20041395$

Anil Kumar, Michaela Knapp-mohammady, P. C. Mishra, SAr Ndor Suhai (2004). A Theoretical Study of Structures and Electron Affinities of Radical Anions of Guanine-Cytosine, Adenine-Thymine, and Hypoxanthine-Cytosine Base Pairs. Journal of Computational Chemistry-25.-1047-1059.DOI: 10.1002/jcc.20020

J. R.Wiley, J. M.Robinson, S.Ehdaie, E. C. M.Chen, E. S. D.Chen, W. E. Wentworth (1991).The determination of absolute electron affinities of the purines and pyrimidines in DNA and RNA from reversible reduction potentials.Biochemical and biophysical research communications.180. $-841-845$. 
E. C. M.Chen, E. S. D.Chen,N.Sane (1998). The electron affinities of the radicals formed by the loss of an aromatic hydrogen atom from adenine, guanine, cytosine, uracil, and thymine. Biochemical and biophysical research communications.-246. - 228-230.

\section{DOI: $10.1006 /$ bbrc. 1998.8584}

E. C. M.Chen, E. S. D.Chen (2000). Negative Ion Mass Spectra, Electron Affinities, Gas Phase Acidities, Bond Dissociation Energies, and Negative Ion States of Cytosine and Thymine.Journal of Physical Chemistry.B.- 104.-7835-7844.DOI: 10.1021/jp001553z

J. Schiedt, R. Weinkauf, D. M. Neumark, E. W. Schlag (1998). Anion spectroscopy of uracil, thymine and the amino-oxo and amino-hydroxytautomers of cytosine and their water clusters. Chemical Physics.-239. -511-524.DOI: 10.1016/S0301-0104(98)00361-9

Kachkovskiy A.D. (1997). The nature of electronic transitions in linearconjugated systems. Russian Chemical Reviews-66(8).-647-664.DOI: 10.1070/RC1997v066n08ABEH000274

M. J. Frisch, G. W. Trucks, H. B. Schlegel, G. E. Scuseria,M. A. Robb, J. R. Cheeseman, J. A. Jr Montgomery, T. Vreven, K. N. Kudin, J. C. Burant, M. Millam, S. S. Iyengar, J. Tomasi, V. Barone, B. Mennucci, M. Cossi, G. Scalmani, N. Rega, G. A. Petersson, H. Nakatsuji, M. Hada, M. Ehara, K. Toyota, R.Fukuda, J. Hasegawa, M. Ishida., T. Nakajima, Y. Honda, O.Kitao, H. Nakai, M. Klene, X. Li, J. E. Knox, H. P. Hratchian, J. B. Cross, C. Adamo, J. Jaramillo, R. Gomperts, R. E. Stratmann, O. Yazyev, A. J. Austin, R. Cammi, C. Pomelli, J. W. Ochterski, P. Y. Ayala, K. Morokuma, G. A. Voth, P. Salvador, J.J Dannenberg, V. G. Zakrzewski, S. Dapprich, A. D. Daniels, M. C. Strain, O. Farkas, D. K. Malick, A. D. Rabuck, K.Raghavachari, J. B. Foresman, J. V. Ortiz, Q. Cui, A. G. Baboul, S. Clifford, J. Cioslowski, B. B. Stefanov, G. Liu, A. Liashenko, P. Piskorz, I. Komaromi, R. L. Martin, D. J. Fox, T.Keith, M. A. Al-Laham, C. Y. Peng, A. Nanayakkara, M.Challacombe, P. M. W. Gill, B. Johnson, W. Chen, M. W. Wong, C. Gonzalez, J. A. Pople, GAUSSIAN03; revision B.05, Gaussian, Inc., Pittsburgh $P A, 2003$.

Pilipchuk N.V., Kachkovski O.D., Yushchenko D.A., Kachkovski G.O. (2005). Electronic properties of polymethine systems 9: position of soliton level in charged molecules. Dyes and Pigments.- 66(3). -223-229. DOI: 10.1016/j.dyepig.2004.06.019

Orlov V. M., Smirnov A.N., VarshavskyYa.M. (1976). Ionization potentials and electron-donor ability of nucleic acid bases and their analogues.Tetrahedron Letters. -48 . $-4377-4378$. 